\title{
Fatores condicionantes na conformação e desempenho das redes de pesquisa fomentadas pelo Ministério da Saúde com foco em saúde coletiva
}

\author{
Conditioning factors in the conformation and performance of research \\ networks promoted by the Ministry of Health with a focus on collective \\ health
}

\section{Factores condicionantes en la conformación y desempeño de las redes de investigación promovidas por el Ministerio de Salud con enfoque en salud colectiva}

Guilherme Arantes Mello ${ }^{1, a}$

gmello@unifesp.br | http://orcid.org/o0oo-0001-5845-6463

\author{
Marge Tenorio ${ }^{2, a}$ \\ marge.tenorio@gmail.com | https://orcid.org/0000-0003-0496-8126
}

Liza Yurie Teruya Uchimura ${ }^{2, a}$

lytuchimura@gmail.com | http://orcid.org/0000-0002-4935-2432

\footnotetext{
${ }^{1}$ Universidade Federal de São Paulo, Escola Paulista de Medicina, Departamento de Medicina Preventiva. São Paulo, SP, Brasil.

2 Ministério da Saúde, Departamento de Ciência e Tecnologia. Brasília, DF, Brasil.

a Doutorado em Medicina Preventiva pela Universidade de São Paulo.
}

\section{RESUMO}

Este artigo apresenta uma análise integrada de três estudos de caso sobre fomentos de redes de pesquisa instituídas pelo Departamento de Ciência e Tecnologia do Ministério da Saúde (Decit), envolvendo três grandes áreas de interesse estratégico nacional: pesquisa clínica, doenças negligenciadas e saúde coletiva. Com foco na saúde coletiva, o estudo desenvolvido, estimulado pelo insucesso do fomento da Rede Nacional de Pesquisas sobre Política de Saúde (RNPPS), busca fundamentar condicionantes que dificultam ou promovem a consolidação de redes de pesquisa nessa área. A capacidade de construção das chamadas, relacionada ao grau de maturidade do campo disciplinar, o modelo de gestão de redes complexas, e o perfil de liderança estão entre os principais condicionantes observados. A par de desafios comuns na consolidação de redes de pesquisa, a saúde coletiva deve aprender a equilibrar com maior propriedade os pesos político e científico na balança do seu campo disciplinar.

Palavras-chave: Saúde coletiva; Projetos de pesquisa; Regionalização; Sistemas de financiamento de pesquisa; Estudo multicêntrico. 


\section{ABSTRACT}

This article presents an integrated analysis of three case studies on the promotion of research networks by the Brazilian Decit - Departamento de Ciência e Tecnologia do Ministério da Saúde (Department of Science and Technology of the Ministry of Health), involving three major areas of national strategic interest: clinical research, neglected diseases and collective health. With the focus on the collective health field, the study seeks to justify the conditions that hinder or promote the consolidation of research networks, and it was stimulated by the failure on the promotion by the RNPPS - Rede Nacional de Pesquisas sobre Política de Saúde (National health policy research network). The capacity for the construction of tjhe formal requests related to the degree of maturity of the study field, the complex network management model, and the leadership profile are among the main conditions observed. With knowledge of common challenges in the consolidation of research networks, the collective health may learn to balance more appropriately the political and scientific weights in the scale of its study field.

Keywords: Collective health field; Research design; Regional health planning; Research financing systems; Multicenter study.

\section{RESUMEN}

Este artículo presenta un análisis integrado de tres estudios de casos sobre el fomento de redes de investigación instituidas por el Decit - Departamento de Ciência e Tecnologia do Ministério da Saúde (Departamento de Ciencia y Tecnología del Ministerio de Salud brasileño), abarcando tres grandes áreas de interés estratégico nacional: investigación clínica, enfermedades olvidadas y salud colectiva. En el caso de la salud colectiva, el estudio fue impulsado por el fracaso del fomento de la RNPPS - Rede Nacional de Pesquisas sobre Política de Saúde (Red nacional de investigaciones sobre políticas de salud) y busca fundamentar los condicionantes que dificultan o promueven la consolidación de redes de investigación. La capacidad de construcción de las convocatorias relacionada al grado de madurez del campo de estudio, modelo de gestión de redes complejas y perfil de liderazgo están entre los principales condicionantes observados. Con el conocimiento de desafíos comunes en la consolidación de redes de investigación, la salud colectiva debe aprender a equilibrar con mayor propiedad los pesos político y científico en la balanza de su campo de estudio.

Palabras clave: Salud colectiva; Proyectos de investigación; Regionalización; Sistemas de financiación para proyetos de investigación; Estudio multicéntrico.

Contribuição dos autores:

Concepção e desenho do estudo: todos os autores.

Aquisição, análise ou interpretação dos dados: todos os autores.

Redação do manuscrito: GAM fez a primeira versão do texto.

Revisão crítica do conteúdo intelectual: todos os autores.

Declaração de conflito de interesses: não há.

Fontes de financiamento: não houve.

Considerações éticas: não há.

Agradecimentos/Contribuições adicionais: a toda equipe do estudo Região e Redes, em particular aos professores Ana Luiza Viana, Nelson Ibañez. Ao prof. Jesús P. Mena-Chalco por sua contribuição essencial com a análise de redes.

Histórico do artigo: submetido: 12 ago. 2019 | aceito: 20 maio 2020 | publicado: 30 set. 2020.

Apresentação anterior: não houve.

Licença CC BY-NC atribuição não comercial. Com essa licença é permitido acessar, baixar (download), copiar, imprimir, compartilhar, reutilizar e distribuir os artigos, desde que para uso não comercial e com a citação da fonte, conferindo os devidos créditos de autoria e menção à Reciis. Nesses casos, nenhuma permissão é necessária por parte dos autores ou dos editores. 


\section{INTRODUÇÃO}

Desde seu despertar nos anos 1970, a saúde coletiva (SC) progressivamente consolida seu campo científico sem nunca esconder a magnitude de suas aspirações: produção de conhecimentos de abrangência interdisciplinar e intersetorial em saúde, especialmente motivada para transformação social. Embora não tenha ainda se definido como ruptura epistêmica essencial ${ }^{1,2}$, a ideia continua pautando o pensamento na área: "a saúde coletiva se consolida como campo científico e âmbito de práticas aberto à incorporação de propostas inovadoras, muito mais do que qualquer outro movimento equivalente na esfera da saúde pública mundial”. Ao envolver campo científico, práticas e propostas inovadoras, esse enunciado revela ambições conceituais e pragmáticas condizentes em outra representação com ideais de ciência, tecnologia e inovação em saúde (CTIS).

Está bem estabelecido o papel estratégico da CTIS para o país. Seja pelo volume do capital internacional envolvido; pela grandeza do saldo negativo da balança comercial desse setor; mas, sobretudo, pela mútua causalidade entre saúde e desenvolvimento ${ }^{3-6}$. Conjuntura que incita a área de saúde coletiva (SC) a se postar no epicentro desse debate. Nesse intuito a SC deve enfrentar o duplo desafio de conferir maior "adensamento conceitual" e "fundamentação teórica" à sua produção científica; enquanto examina caminhos da inovação que assegurem a devida independência tecnológica para saúde brasileira - reconhecidamente sinuosos e pouco definidos9.

Estudos comparativos mostram que a posição brasileira em CTIS avançou, mas ainda se mantém em clara desvantagem competitiva internacional. Endogamia intelectual, aderência acadêmica, fraca interação multiprofissional, e baixa internacionalização são obstáculos comuns no ambiente científico e tecnológico do país ${ }^{10}$. Em última análise, observam-se questões alusivas à dimensão e diversidade das redes intelectuais e de pesquisa do campo disciplinar.

\section{O PAPEL DA POLÍTICA DE FOMENTO ESTRATÉGICO NA CONFORMAÇÃO DA CTIS}

A superação da visão linear da inovação tecnológica esclarece que, embora dependam de incremento financeiro, avanços nesse sistema são fundamentalmente impulsionados por incisiva direcionalidade política, traduzida em agendas estratégicas ${ }^{11,12}$.

No início deste século foi criada nos Estados Unidos uma agressiva política estratégica de fomento denominada Roadmap for medical research, resultante de um processo de consulta a centenas de lideranças acadêmicas, governamentais e do setor privado ${ }^{13}$. Um direcionamento que efetivamente define agendas, prioridades e orienta a reestruturação de processos e investimentos voltados para manutenção da independência tecnológica e liderança internacional dos Estados Unidos em CTIS - dessa agenda deriva, por exemplo, a ideia de 'pesquisa translacional', e a visão de consolidar redes de pesquisas translacionais com a capacitação de milhares de profissionais dos serviços de saúde ${ }^{14-16}$.

Nesse mesmo período as políticas de CTIS também experimentam intensa inflexão no Brasil. Mas, diversa da norte-americana, o maior desafio da realidade brasileira estava em romper com a inércia dos

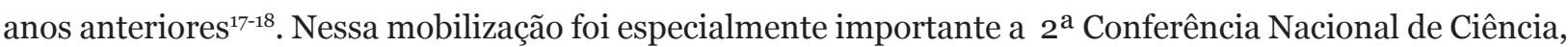
Tecnologia e Inovação em Saúde, em 2004, quando foram aprovadas a Política Nacional de Ciência, Tecnologia e Inovação em Saúde e Agenda Nacional de Prioridades de Pesquisa em Saúde ${ }^{19-20}$ - formatada como um catálogo de temas e questões nas variadas áreas, acabou representando mais um marco simbólico do que verdadeiramente uma agenda, ao se ver descolada de objetivos, metas, prazos e financiamentos específicos. Esse conjunto de articulações é suficiente para definir um novo ciclo produtivo para o sistema nacional de inovação em saúde ${ }^{21}$. 
Todo esse processo serviu de grande aprendizado político, burocrático e técnico para o novo Departamento de Ciência e Tecnologia do Ministério da Saúde (Decit-MS) em seu desígnio de promover pesquisas estratégicas em saúde - a ideia de pesquisa estratégica embute questões não tratadas aqui sobre cenários de desenvolvimento (acadêmicos, institutos, empresas); julgamento do mérito versus relevância; papel da demanda e oferta, entre outras ${ }^{22}$. Desde o início, sua política é despertada para o fomento de redes de pesquisas estratégicas sobre problemas nacionais.

\section{REDES DE PESQUISA}

Já em 2003 o Decit enuncia o propósito de uma Rede de Pesquisa em Dengue, e participa no financiamento da Rede Brasileira de Pesquisas e Combate a Tuberculose - Rede TB, contemplada, desde 2001, no projeto Instituto do Milênio ${ }^{23}$. Com maior aporte de recursos, esses ideais são fortalecidos no ano de 2004, quando se define a "transição dos fomentos imaturos". Contudo, a intenção de se firmar redes de pesquisa esbarra na ausência de um modelo definido de integração, na fragmentação e em uma baixa identidade entre os grupos de pesquisa. Além disso, resultados esperados em médio e longo prazo já prediziam a prolongada trajetória de aprendizado deste tipo de indução.

Seguindo outras iniciativas, em 2014 é realizada uma chamada no campo da saúde coletiva com o objetivo de consolidar a Rede Nacional de Pesquisas sobre Política de Saúde (RNPPS), MCTI/CNPq/CT - Saúde/MS/SCTIE/Decit No 41/2013 ${ }^{24}$ prevista na Portaria 193/GM/MS ${ }^{25}$. Conquanto o esforço tenha gerado vários produtos, a conformação da RNPPS efetivamente não se concretizou.

Despertado pelo interesse no campo da SC e, em particular, na RNPPS, este estudo discute condicionantes do fomento estratégico de redes de pesquisa e estudos multicêntricos a partir de três experiências de fomento estratégico realizadas pelo Decit.

\section{MÉTODO}

Análise integrada de três estudos de caso sobre redes de pesquisas estratégicas fomentadas pelo Decit em três campos estratégicos: pesquisa clínica, doenças negligenciadas e saúde coletiva:

1. Chamada de fomento nacional MCT/CNPq/MS-SCTIE/Decit No 25/2006, 'Avaliação da Política de Ciência, Tecnologia e Inovação em Saúde Implementada pelo Departamento de Ciência e Tecnologia do Ministério da Saúde, no período de 2003 a 2010'. Subprojeto 'Desenvolvimento de novas abordagens para o controle de Aedes aegypti, vetor de dengue no Brasil'. Estudo de caso fundamentado em dados, realizado entre 2012-13. A principal fonte de evidências consistiu em entrevistas com lideranças do Decit, Conselho Nacional de Desenvolvimento Científico e Tecnológico (CNPq) e científicas, num total de 23, espalhadas por sete estados (10 cidades), e uma no exterior. Os resultados foram apresentados ao Decit e em seminário específico do projeto.

2. Chamada de fomento nacional MCT/Finep/MS/SCTIE/Decit ação transversal - Implantação de Unidades de Pesquisa Clínica - 04/2005. Iniciativa dos Ministérios da Saúde e da Ciência e Tecnologia direcionada à conformação da Rede Nacional de Pesquisa Clínica (RNPC) em Hospitais de Ensino ${ }^{26}$. Este estudo de caso resultou em uma tese de doutorado ${ }^{27}$ e três artigos específicos ${ }^{28,29}$.

3. Chamada de fomento nacional MCTI/CNPq/CT - Saúde/MS/SCTIE/Decit No 41/2013. Linha de pesquisa $\mathrm{n}^{0} 5$ : 'Identificação e análise de iniciativas inovadoras de melhoria da efetividade e da eficiência dos processos da coordenação e organização das redes assistenciais e regiões de saúde e seu impacto nos fluxos de demanda e estruturação da oferta'. Especificamente sobre o subprojeto contemplado: Projeto 'Política, Planejamento e Gestão das Regiões e Redes de Atenção à Saúde no 
Brasil ('Região e Redes')'. Estudo realizado especificamente para este artigo, em triangulação com a produção técnica-científica do projeto; dinâmica de gestão e experiência dos autores sobre categorias analíticas dos demais estudos.

Conforme detalhado por Mena-Chalco ${ }^{30}$, na geração das redes de coautorias o software scriptLattes foi utilizado para identificar a produção acadêmica e vínculos de cooperação dos pesquisadores selecionados no Currículo Lattes (sujeito à atualização dos currículos). Os dados foram organizados em banco de dados e o desenho das matrizes de coautorias construído com o auxílio do UCINET software for social network analysis.

\section{RESULTADOS}

\section{Um longo aprendizado}

A aspiração de fomentar redes de pesquisa encontrou sua primeira dificuldade no próprio conceito produtivo. Como rememora uma pesquisadora envolvida naquele início da Rede Dengue sobre encontro específico promovido pela Fiocruz para o incentivo de redes de pesquisa: "E alguém perguntou: o que era uma rede? Ninguém sabia responder, inclusive as pessoas que estavam organizando [risos]. Então ficou claro que ninguém sabia ali o que é que era uma rede" ${ }^{11}$. Assim, teoria e prática teriam que maturar em paralelo nas várias redes financiadas (Quadro 1).

Quadro 1-Redes de pesquisas instituídas pelo Decit/SCTIE/MS, 2003 - 2016

\begin{tabular}{|c|c|c|}
\hline Ano & Identificação da rede & $\begin{array}{c}\text { Área de } \\
\text { concentração }\end{array}$ \\
\hline 2003 & Rede TB & Saúde pública \\
\hline 2004 & Rede Dengue & Saúde pública \\
\hline \multirow{2}{*}{2005} & Rede Nacional de Pesquisa Clínica (RNPC) & Pesquisa clínica \\
\hline & Rede Multicêntrica de Avaliação de Implantes Ortopédicos (REMATO) & Tecnológica \\
\hline 2006 & Rede Nordeste de Biotecnologia (RENORBIO) & Biomédica \\
\hline \multirow{2}{*}{2008} & Rede Nacional de Terapia Celular (RNTC) & Biomédica \\
\hline & Rede Câncer & Pesquisa clínica \\
\hline \multirow{3}{*}{2009} & Rede Dengue & Saúde pública \\
\hline & Rede Malária & Saúde pública \\
\hline & Rede Brasileira de Avaliação de Tecnologia em Saúde (REBRATS) & Tecnológica \\
\hline \multirow{5}{*}{2013} & Rede Nacional de Pesquisa em Acidente Vascular Cerebral (RNPAVC) & Pesquisa clínica \\
\hline & Rede Nacional de Pesquisas sobre Política de Saúde (RNPPS) & Saúde pública \\
\hline & Rede Nacional de Pesquisa Clínica em Câncer (RNPCC) & Pesquisa clínica \\
\hline & Rede Nacional de Pesquisa em Doenças Cardiovasculares (RNPDC) & Pesquisa clínica \\
\hline & Rede Nacional de Pesquisas em Doenças Negligenciadas (RNPDN) & Pesquisa clínica \\
\hline 2016 & Rede Nacional de Especialistas em Zika e Doenças Correlatas (RENEZIKA) & Saúde pública \\
\hline
\end{tabular}

Fonte: Decit/SCTIE/MS (2019).

O ensaio primordial do Decit com este intento consistiu na aprovação de 13 projetos cooperativos da Rede Dengue em 2003/4. Havia a expectativa de que a aproximação desses grupos poderia despertar 
interações que desembocassem em colaborações em rede ${ }^{23}$, ideia rapidamente desfeita. No edital de 2006 foi estabelecida lógica diversa, com a determinação do comitê de avaliação da primeira fase para que projetos de 23 grupos nacionais fossem reunidos sob coordenação unificada do proponente da Fiocruz-RJ. Além da integração da rede, também visava minorar duplicidades de financiamento e estabelecer padronizações de rotinas para efeito de comparabilidade. A destinação de $\mathrm{R} \$$ 800.000,00 foi expressiva para aquele momento, mas representava uma redução de $60 \%$ na soma dos orçamentos originais. Esse déficit, somado ao constrangimento da subordinação involuntária a uma coordenação central, e ao enorme acréscimo de burocracia interestadual, gerou uma experiência fortemente traumática entre os coordenadores dos subprojetos.

O estímulo não foi ainda suficiente para integração de objetivos e efetivação da Rede Dengue, mas alguns ganhos, que provavelmente não ocorreriam com os projetos isolados, foram notáveis: os objetivos originais de cada grupo não foram prejudicados (senão por cortes que provavelmente os afetariam de todo modo); e o grupo maior teve suas relações profissionais, comunicação científica e identidade nacional nitidamente fortalecidas, refletindo em ganho de autonomia do campo da dengue no país. Percepção retrospectiva e externa que provavelmente ainda contrasta com penosa experiência pessoal das coordenações. Contudo, sensações contraditórias desses ganhos já eram experimentadas pelos laboratórios menores: "Se você acredita naqueles casamentos à moda antiga, que o casamento vem primeiro e o amor vem depois, aí pode ser... [...] Mas, assim, é claro que foi bom para mim [...] Então, para os grupos que são emergentes... favorece sim"31. Embora o estudo não tenha se estendido sobre o desenvolvimento posterior da Rede Dengue, a chamada Pronexdengue de 2009, na qual o volume de financiamento salta para $\mathrm{R}$ \$ 10 milhões, é notavelmente cingida pelos grupos do edital anterior, apontando para o amadurecimento de um conjunto de atores interligados aptos a financiamentos maiores. Alguns coordenadores do edital de 2006 foram questionados sobre a ideia de se repetir a estratégia de 2006, reunindo alguns grupos dentro do Pronex-dengue. A possibilidade foi refutada de modo firme e unânime como contrassenso inaceitável naquele estágio.

\section{O desafio dos fomentos maduros}

Despertada em 2005, a RNPC significou um grande esforço de investimento interministerial. Complementando o valor inicial de R $\$ 35$ milhões para estruturação, outras cinco chamadas objetivaram o desenvolvimento de estudos multicêntricos (editais em 2007, 2008, 2010 e dois em 2013), totalizando R \$ 68 milhões (empenhados $\mathrm{R} \$ 54,3$ milhões em 55 projetos) ${ }^{29,32}$.

Com notável dimensão e complexidade, a RNPC foi integrada pela reunião colaborativa de Unidades de Pesquisa Clínica (UPC), que de acordo com suas características se definiam como sub-redes autônomas. No exemplo mais visível, a Fiocruz compõe a RNPC com sua própria 'Rede Fiocruz de Pesquisa Clínica' (6o grupos): ao mesmo tempo UPC da RNPC e rede independente. Para essa integração foi estabelecido um Comitê Gestor (Ministérios da Saúde e Ciência e Tecnologia, Finep, CNPq); e um Centro Coordenador (uma UPC apoiada por quatro grupos de trabalho) com a responsabilidade de articulação das sub-redes.

Em si, esse arranjo revelou-se insuficiente para estabilidade da rede e efetivação de parcerias multicêntricas. Entre os principais problemas operacionais, foram encontradas dificuldades para superar uma gestão verticalizada e hierárquica em direção a um processo poliárquico e colaborativo; reuniões de coordenação esvaziadas; falhas no monitoramento e registros dos projetos - apenas cerca de $15 \%$ dos mais de 700 projetos de pesquisa clínica das UCP estavam sob monitoramento; e carência de instrumentos integradores - a rede sequer contava com uma página de internet. Fatores decisivos para minar a identidade, entusiasmo e continuidade de ações ${ }^{19}$. Como resultado, parceiros externos referiram dificuldade em identificar a RNPC, senão "centros individuais" rotulados como rede $\mathrm{d}^{20,28,29}$. 
Mas, a realidade é que o esforço de gestão foi fortemente prejudicado por falhas na concepção dos editais, que impactaram todo o processo. Especialmente ao deixarem de exigir organização multicêntrica das submissões; parcerias entre as UPC; e exclusividade para estudos clínicos. Também ao permitir financiamento externo à rede ${ }^{29}$.

Na conformação da RNPPS, o problema da construção da chamada revelou-se contraproducente em particular. O distanciamento entre as linhas de pesquisa estipuladas, fruto de excessiva ingerência política, já permitia antever que estas chamadas funcionariam como editais isolados. Magnificada pela diversidade de propósitos em um campo de grandes dimensões políticas, a própria definição da coordenação geral da rede se vaticinou inviabilizada.

Como linha independente, o projeto Região e Redes buscou franco apoio na experiência da RNPC. Com objetivos geral e específicos integrados, foram reunidos diversos grupos autônomos com suas próprias redes independentes de colaboração (unidades de pesquisa denominadas 'dimensões'). Com isto foi conformada uma rede multicêntrica com envolvimento direto de 18 instituições e 132 pesquisadores das cinco regiões brasileiras ${ }^{13,33}$. Como na RNPC, um típico modelamento de redes complexas inseridas em redes complexas. Como aquela, o modelo de coordenação foi organizado sobre uma unidade gestora; conselho de pesquisa; e comitê guia para cada dimensão (sub-redes). Duas ferramentas, que compõem categoria de análise própria, mostraram-se indispensáveis ao esforço sustentado de colaboração poliárquica e criativa: um website; e rede social dos pesquisadores envolvendo $e$-mail e WhatsApp.

\section{Análise da produtividade do fomento}

Segundo o relatório da pesquisa, do edital da Rede Dengue de 2006 originaram nove mestrados; dez doutorados; vários artigos científicos; proposição de novos indicadores epidemiológicos em dengue; sistematização de monitoramento de uso e resistência de inseticidas; alternativas de controle vetorial, entre outros. Estimativa compreensível e importante para um relatório final, mas certamente um tanto imprecisa ao envolver processos usualmente mais demorados do que os prazos dos editais; e em boa parte com financiamentos sobrepostos: "Então este produto foi resultado do primeiro financiamento do Decit [2004], mais PDTSP, mais uma parte do projeto 'saudável', que era financiado pela Finep e pelo CNPq”. Ademais, alguns dos principais frutos somente podem ser estimados anos depois, num misto de resultados palpáveis e subjetivos, como revela uma coordenadora de laboratório cerca de quatro anos depois do término do edital: "ele teve uma série de desdobramentos que eu acho que só foi possível graças a este financiamento inicial, que a gente está na verdade colhendo os frutos somente agora" ${ }^{31}$. Não seria exagero assumir que, para os grupos menores, o ganho essencial foi a própria oportunidade de subsistência e integração ao campo disciplinar (produtos que se tornam visíveis apenas com o passar dos anos).

Contrariando as expectativas projetadas, o vultoso investimento na RNPC não se refletiu em produtos mais sistemáticos: "essa estrutura não funcionou efetivamente no que diz respeito à interação dos grupos de trabalho e geração de produtos ou de qualquer outro resultado" 28 .

A preocupação com a latência da produção dos estudos multicêntricos levou o estudo Região e Redes a definir produtos intermediários sistemáticos para as sub-redes na forma de relatórios, notas técnicas e dossiês. Estratégia que se mostrou produtiva em vários sentidos: como meio de divulgação, atualização e intercâmbio sistematizado de informações entre os diversos grupos envolvidos; capacidade de divulgação precoce dos resultados; e, especificamente, como base para produção de artigos científicos, publicados ainda no decorrer do edital (Quadro 2). 
Quadro 2 - Produção técnica e científica da pesquisa Região e Redes, 2014-2018

\begin{tabular}{|l|l|}
\hline Tipo de produção bibliográfica & N \\
\hline Dossiê regiões e redes & 05 \\
\hline Notas técnicas & 16 \\
\hline Artigos científicos & 24 \\
\hline Teses e dissertações concluídas & 04 \\
\hline Teses e dissertações em andamento & 06 \\
\hline Estudos com indicadores regionais & 04 \\
\hline Banco de indicadores regionais e tipologias & 01 \\
\hline
\end{tabular}

Fonte: Elaboração própria (2019).

A interação das sub-redes do projeto teve ampla continência, traduzida na integração de objetivos comuns e complementares e, finalmente, no processo de colaboração dos produtos (Figura 1 e 2). Assumidamente influenciada por essa interação, pelo menos uma sub-rede de projeção nacional reorientou suas análises dos municípios para regiões.

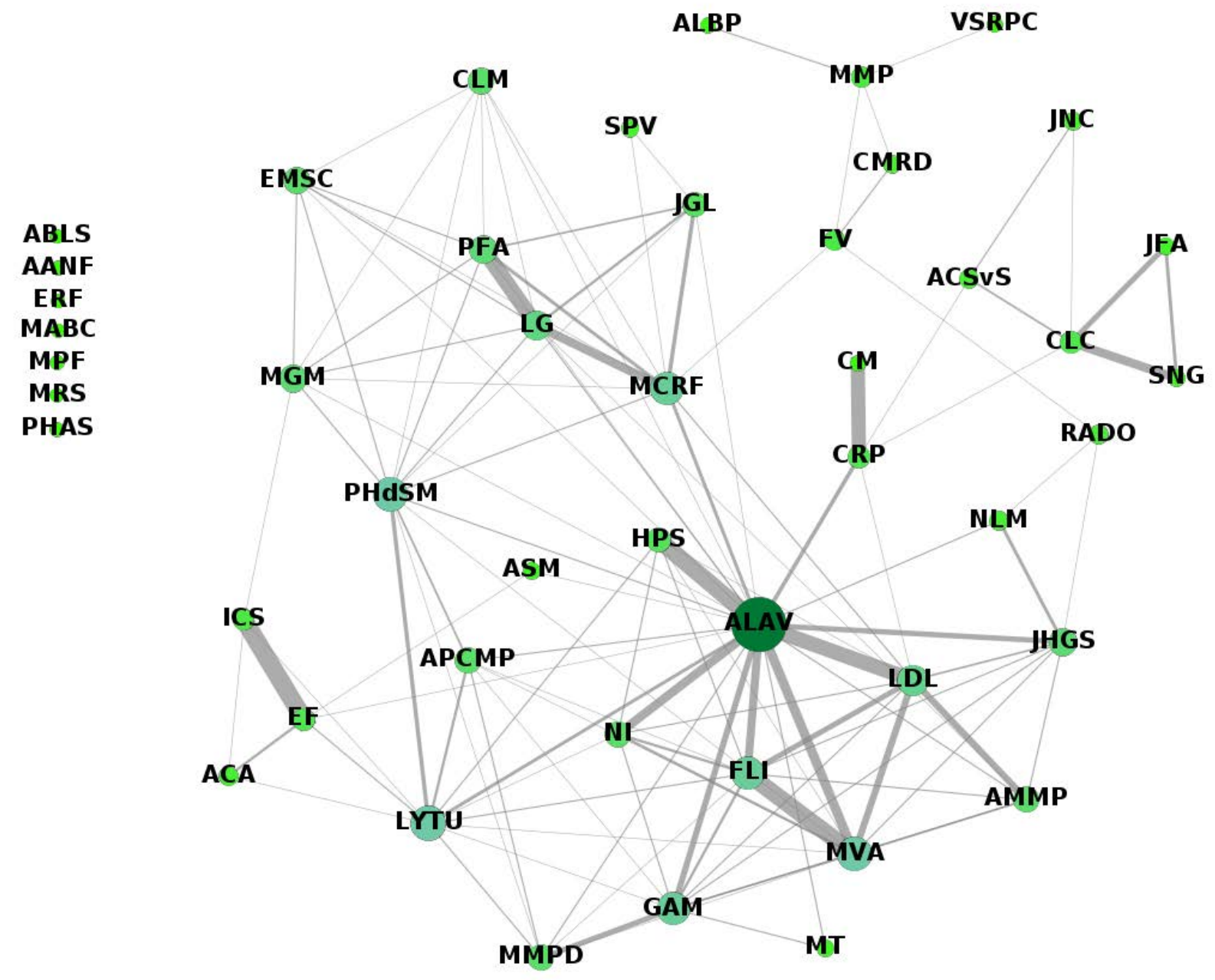

Figura 1 - Rede de coautorias entre pesquisadores da pesquisa Região e Redes, 2014-2017

Fonte: Plataforma Lattes (2018). 

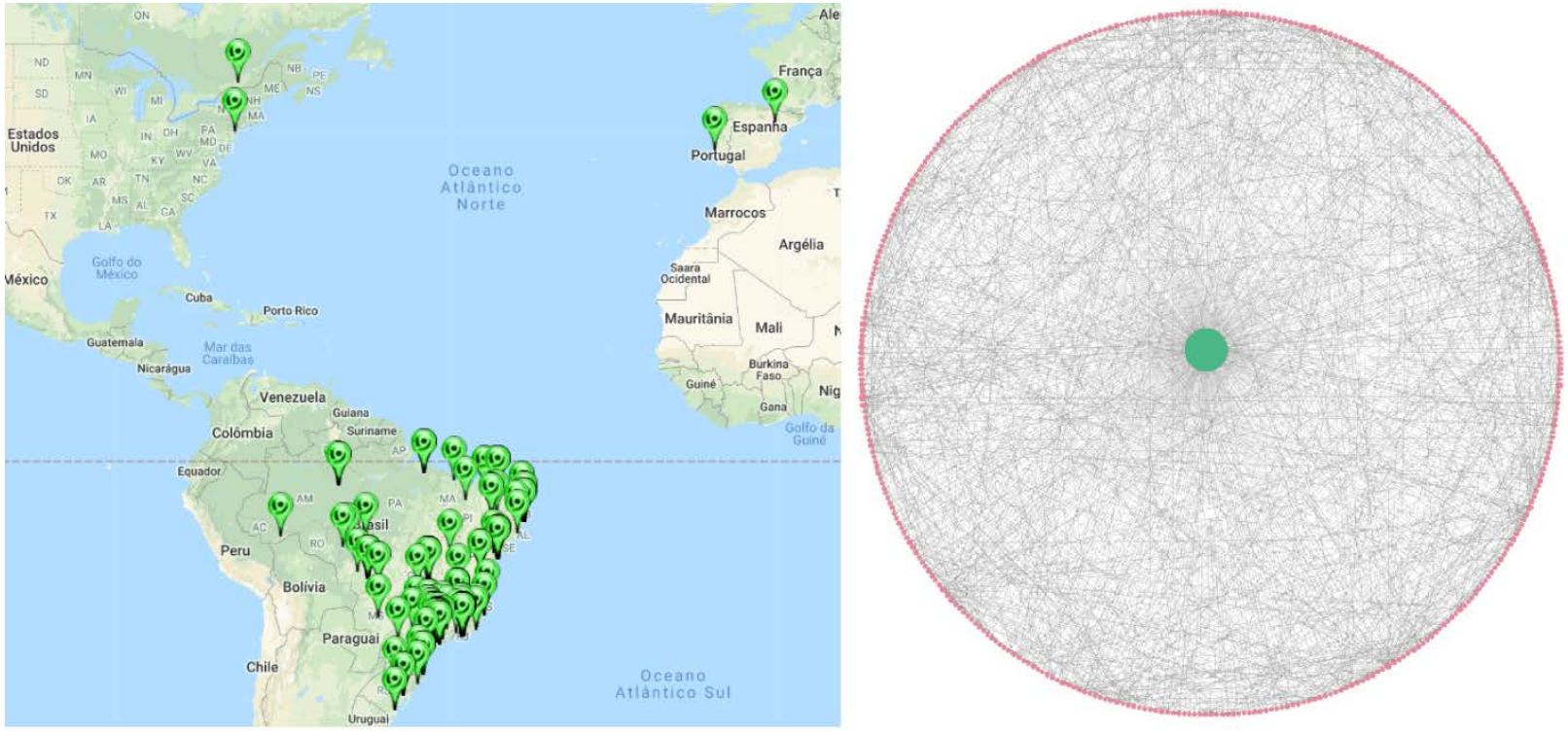

Figura 2 - Geolocalização e grafo da rede expandida de coautorias da pesquisa Região e Redes 2014-2017. Os pesquisadores do projeto estão agrupados no círculo central e os 389 vértices periféricos representam colaboradores Fonte: Plataforma Lattes (2018).

\section{Disseminação dos resultados na era da informação}

No geral, as duas primeiras redes seguiram os caminhos clássicos de 'comunicação', 'disseminação' e ‘divulgação’ científica de seus produtos ${ }^{34,35}$, caminhos reconhecidamente laboriosos. Fruto de seu tempo, o estudo Região e Redes desenvolveu estratégia original, ao integrar um núcleo de comunicação profissional ao projeto.

Com isso foram testados novos meios e desenvolvidas redes sociais sistematizadas para disseminação e interação sobre o andamento e resultados da pesquisa. Página oficial na internet, rede social no Facebook, e perfis no Youtube e Flickr foram utilizadas como plataformas de informações, permitindo hospedar diferentes formatos de conteúdos e produtos adaptáveis à escolha individualizada do leitor.

Análises de monitoramento permitiram reorganizar estratégias de divulgação científica em massa, resultando em categórica interiorização e internacionalização do conhecimento gerado. No país, São Paulo e Rio de Janeiro, maiores centros urbanos e acadêmicos, representaram apenas cerca de 30\% do público da pesquisa, com o restante de seguidores capilarizados por todo território nacional (Quadro 3). A organização de um seminário internacional em São Paulo sobre resultados preliminares contribuiu para consolidar a divulgação entre pesquisadores e gestores de vários estados.

\section{Quadro 3 - Número de seguidores no Facebook da pesquisa Região e Redes distribuídos por país, cidade e idioma, 2014-2017}

(continua)

\begin{tabular}{|l|c|}
\hline \multicolumn{1}{|c|}{ País } & n \\
\hline Brasil & 196.355 \\
\hline Estados Unidos & 1054 \\
\hline Portugal & 787 \\
\hline Itália & 349 \\
\hline Espanha & 330 \\
\hline Reino Unido & 319 \\
\hline
\end{tabular}

\begin{tabular}{|l|c|}
\hline \multicolumn{1}{|c|}{ Cidades brasileiras } & n \\
\hline São Paulo (SP) & 18.985 \\
\hline Rio de Janeiro (RJ) & 11.538 \\
\hline Salvador (BA) & 5.356 \\
\hline Porto Alegre (RS) & 4.372 \\
\hline Brasília (DF) & 4.355 \\
\hline Curitiba (PR) & 3.943 \\
\hline
\end{tabular}

\begin{tabular}{|l|c|}
\hline \multicolumn{1}{|c|}{ Idioma } & n \\
\hline Português (Brasil) & 192.349 \\
\hline Português (Portugal) & 3.840 \\
\hline Inglês (EUA) & 2.895 \\
\hline Espanhol & 959 \\
\hline Inglês (Reino Unido) & 753 \\
\hline Francês & 509 \\
\hline
\end{tabular}


(conclusão)

\begin{tabular}{|c|c|c|c|}
\hline País & $\mathbf{n}$ & Cidades brasileiras & $\mathbf{n}$ \\
\hline França & 316 & Fortaleza (CE) & 3.873 \\
\hline Argentina & 292 & Recife (PE) & 3.842 \\
\hline Alemanha & 282 & Campinas (SP) & 3.508 \\
\hline Canadá & 277 & Belo Horizonte (MG) & 3.498 \\
\hline Austrália & 150 & Manaus (AM) & 3.056 \\
\hline Japão & 149 & Belém (PA) & 3.014 \\
\hline México & 119 & Florianópolis (SC) & 2.012 \\
\hline Chile & 118 & Santa Maria (RS) & 1.546 \\
\hline Suíça & 108 & Natal (RN) & 1.522 \\
\hline Holanda & 103 & Campo Grande (MS) & 1.404 \\
\hline Irlanda & 100 & Niterói (RJ) & 1.364 \\
\hline Peru & 88 & Goiânia (GO) & 1.248 \\
\hline Uruguai & 82 & São Carlos (SP) & 1.230 \\
\hline Paraguai & 81 & Juiz de Fora (MG) & 1.220 \\
\hline Bolívia & 74 & Aracaju (SE) & 1.210 \\
\hline Colômbia & 72 & Pelotas (RS) & 1.203 \\
\hline Angola & 51 & João Pessoa (PB) & 1.190 \\
\hline Bélgica & 50 & Teresina (PI) & 1.137 \\
\hline Índia & 40 & Maceió (AL) & 1.065 \\
\hline Suécia & 40 & São Luis (MA) & 1.006 \\
\hline Nova Zelândia & 31 & Cuiabá (MT) & 1.004 \\
\hline Equador & 27 & Ribeirão Preto (SP) & 918 \\
\hline Áustria & 25 & Santo André (SP) & 875 \\
\hline Israel & 22 & Santos (SP) & 870 \\
\hline Turquia & 22 & $\begin{array}{l}\text { São José dos Campos } \\
\text { (SP) }\end{array}$ & 858 \\
\hline Noruega & 22 & Jundiaí (SP) & 700 \\
\hline Cuba & 19 & Guarulhos (SP) & 695 \\
\hline Egito & 19 & Maringá (PR) & 624 \\
\hline Moçambique & 19 & Uberlândia (MG) & 623 \\
\hline China & 17 & Caruaru (PE) & 622 \\
\hline Dinamarca & 17 & Sorocaba (SP) & 610 \\
\hline Argélia & 17 & Chapecó (SC) & 600 \\
\hline Cabo Verde & 16 & Palmas (TO) & 607 \\
\hline Emirados Árabes Unidos & 16 & Montes Claros (MG) & 600 \\
\hline Polônia & 15 & Porto Velho (RO) & 600 \\
\hline Filipinas & 15 & Londrina (PR) & 569 \\
\hline Finlândia & 14 & São Gonçalo (RJ) & 582 \\
\hline África do Sul & 14 & & \\
\hline Guatemala & 14 & & \\
\hline
\end{tabular}

\begin{tabular}{|l|c|}
\hline \multicolumn{1}{|c|}{ Idioma } & $\mathbf{n}$ \\
\hline Espanhol (Espanha) & 433 \\
\hline Italiano & 309 \\
\hline Alemão & 188 \\
\hline Árabe & 31 \\
\hline Japonês & 30 \\
\hline Holandês & 21 \\
\hline Francês (Canadá) & 17 \\
\hline Turco & 15 \\
\hline Chinês & \\
\hline & \\
\hline & \\
\hline & \\
\hline & \\
\hline & \\
\hline & \\
\hline & \\
\hline & \\
\hline & \\
\hline & \\
\hline & \\
\hline & \\
\hline & \\
\hline & \\
\hline & \\
\hline & \\
\hline & \\
\hline & \\
\hline & \\
\hline & \\
\hline & \\
\hline & \\
\hline & \\
\hline & \\
\hline & \\
\hline & \\
\hline & \\
\hline & \\
\hline & \\
\hline & \\
\hline & \\
\hline & \\
\hline & \\
\hline & \\
\hline & \\
\hline & \\
\hline & \\
\hline & \\
\hline & \\
\hline & \\
\hline & \\
\hline & \\
\hline & \\
\hline & \\
\hline & \\
\hline & \\
\hline & \\
\hline & \\
\hline & \\
\hline & \\
\hline & \\
\hline & \\
\hline
\end{tabular}

Fonte: Elaboração própria (2019) .

Estimulado, o componente de comunicação ganhou autonomia a ponto de se comportar propriamente como mídia. Isto é, deixar a posição passiva de espera de resultados da pesquisa, para uma posição ativa 
de coprodução desse conteúdo - o que o retira da perspectiva de ferramenta para componente próprio da investigação. Disto originaram-se, por exemplo, inúmeras entrevistas com personalidades do campo e divulgação de materiais correlatos.

\section{A LIDERANÇA COMO PAPEL VITAL}

O perfil da liderança da rede surge como categoria original (não contemplada como pré-categoria) marcante nos três estudos. Apesar do enorme desconforto com o edital de 2006, as citações sobre a coordenação geral foram permeadas por respeito e consideração; dispondo-a como responsável basilar pela motivação, unidade e colaboração entre os grupos. De igual maneira ocorreu no projeto Região e Redes. Exigida em outra escala de complexidade, as dificuldades com liderança estiveram entre os fatores centrais de retração da RNPC.

\section{DISCUSSÃO}

A capacidade de construção dos editais mostrou-se decisiva para efetividade do fomento e certamente está relacionada com a experiência e maturidade do campo fomentado. A simples reunião de projetos no julgamento do edital visando às interações em rede apresenta limitado alcance, como revelado no edital de dengue de 2006 - opção possível de ser aventada para campos imaturos, mas bem pesada a possibilidade de efeitos colaterais, e capacidade de liderança da coordenação geral. Essa limitação é corroborada por experiência semelhante da Fiocruz no desenvolvimento do Programa de Desenvolvimento e Inovação Tecnológica em Saúde Pública (PDTSP) ${ }^{36}$. Desse aprendizado resultou uma síntese transformada em aforisma no âmbito do fomento: 'rede de pesquisa não significa coleção de projetos'.

O modelo almejado passa então a se definir como voltado para investigações multicêntricas com objetivos gerais ambiciosos; e objetivos específicos integrados entre grupos interdisciplinares. Para campos imaturos, uma rede de pesquisa disciplinar pode começar a se constituir a partir de projeto multicêntrico (e estudos multicêntricos muito grandes podem representar uma própria rede disciplinar).

'Rede de pesquisa' e 'pesquisa em rede' se confundem nessa discussão. Em termos gerais, a primeira representa o tecido de suporte e integração de várias pesquisas e grupos no campo disciplinar. A pesquisa em rede - multicêntrica - representa uma sub-rede organizada com objetivos próprios. Quanto mais projetos e variabilidade dos grupos, mais sólida e inovadora a rede de pesquisa ${ }^{37}$; maior sua competência na advocacy para que projetos multicêntricos não encerrem em evento único - como de fato ocorreu com a pesquisa Região e Redes sem a RNPPS. É certo que a consolidação de redes de pesquisa exige financiamentos progressivos para lidar com o crescimento, competências e complexidade adquiridas, o que seguramente reivindica em determinado ponto a superação do excessivo esforço burocrático e a flutuação típicos da cultura de editais. Mas não apenas.

O perfil de liderança emerge neste estudo como categoria analítica central para a estabilidade dessas redes. Sem dominar todos os referenciais envolvidos - e, talvez mesmo não ser a mais graduada, a coordenação geral deve ser reconhecida pelos subgrupos por sua capacidade científica, administrativa e política, o que exige altas doses de diplomacia. No caso das redes, um desafio particular às lideranças figura na contradição existente no fato de estabelecer identidade, objetivos, pertencimento e coesão para rede multicêntrica, ao mesmo tempo em que se lida e estimula a identidade, autonomia, incertezas e criatividade das sub-redes autônomas. Cada uma com suas características de intermediações, centralidades e agrupamentos, em um enovelamento de redes complexas multiescalares que operam em constante equilíbrio dinâmico, pressionadas por incertezas e imprevisibilidades (embora referenciais específicos venham sendo introduzidos na área da saúde, sua compreensão e aplicabilidades são ainda incertos ${ }^{38}$ ). 
A formação dessas lideranças representa um desafio institucional altamente trabalhoso e delicado: graduação sólida com experiência em iniciação científica e política estudantil; formação com mestrado, doutorado, pós-doutorado e desenvolvimento profissional em diferentes grupos e instituições; experiência de ensino; participação e coordenação de programas de pós-graduação; organização e liderança de laboratórios e equipes; participação e organização de encontros e congressos, entre outras características. O tempo é fator insuperável nessa formação - e pode haver quebra definitiva deste ciclo a qualquer instante, por fatores pessoais, institucionais ou, em especial, de estabilidade política. Disso deduz-se que experiências multicêntricas propiciam oportunidades valiosíssimas de aprendizado científico.

A estratégia de comunicação surge como propriedade intrínseca à capacidade de gestão da rede, que pode ser dividida entre comunicação interna (rede) e externa (científica e social). A dificuldade de comunicação interna sobressaiu-se como um nó górdio para a RNPC, com baixa atenção a registros; ineficiência de informação, comunicação e reconhecimento de afinidades e capacidades entre as UPC. As tecnologias disponíveis foram subutilizadas e a RNPC sequer desenvolveu uma identidade virtual própria. Sobre esse aprendizado, a organização profissional da comunicação se revelou uma inovação valorosa para identidade e coesão de objetivos na rede estabelecida pelo projeto Região e Redes. A interação social virtual e encontros - mobilizada entre os pesquisadores através do WhatsApp desempenhou importante papel na sensação de pertencimento, o que é descrito como "manejo de interação social" da rede ${ }^{39}$.

No que se refere ao ambiente externo, a premência de resultados não é exclusividade da SC, mas as diferenças entre tempo acadêmico e tempo político é particularmente sensível neste campo. Além disso, em contraste com pesquisa clínica e de bancada, na SC é mais plausível a liberação, para o debate, de materiais tecnicamente menos aprimorados. Sobre essas características, a profissionalização da comunicação da pesquisa mostrou-se efetiva ao aproveitar conhecimentos já estabelecidos em outras áreas, mas ainda pouco utilizados no campo científico. Com isso, as redes virtuais desenvolvidas permitiram contornar barreiras de difusão tradicionais, como necessidade de consentimentos específicos e intermediação de atores-chave. Além disso, os vários tipos de canais de comunicação e formatos de produtos permitiram atingir públicos diferenciados em grande abrangência geográfica.

Apesar dessa constatação, a avaliação mais específica da efetividade do fomento de redes surge como problema particular. Análises bibliométricas tradicionais fornecem importantes evidências sobre o crescimento e influência no campo científico estimulado, embora sujeitas a interpretações, manipulações e baixa identificação com as necessidades estratégicas regionais ${ }^{40-43}$. Falta clareza sobre indicadores de redes propriamente - a representação de coautorias tem grande apelo visual, mas ainda se encontra em definição sobre seu real valor ${ }^{44,45}$. Ambos falham em superar o componente 'ciência' e, particularmente cobiçados, indicadores tecnológicos estão ainda menos sistematizados.

\section{CONCLUSÕES}

Como considerações gerais, o longo aprendizado do Decit revela que, para efetividade do fomento de redes estratégicas de pesquisa, é determinante uma forte intencionalidade política atrelada a uma agenda estratégica que defina os objetivos, metas, prazos e financiamentos estáveis. Deixa claro ainda o papel decisivo dessa estabilidade para formação de lideranças científicas.

Especificamente sobre a conformação de redes de pesquisa e pesquisas multicêntricas, os estudos revisados evidenciam um conjunto de obstáculos passíveis de ser contornados: capacidade de construção da chamada, modelo de gestão de redes complexas, estratégias de comunicação e perfil de liderança estão entre os principais condicionantes de êxito das redes multicêntricas - questões também relacionadas com o grau de maturidade do campo disciplinar. 
Este estudo apresenta limitações ao revisar trabalhos desenvolvidos em momentos diversos e com objetivos independentes, de forma que nem todos disponibilizavam as mesmas categorias de análise ou a mesma abrangência. Sua validade externa é limitada pelo método qualitativo e intencionalidade da amostra.

Sobre fomento científico, esta revisão deixa patente a baixa disponibilidade de estudos específicos na literatura brasileira, um agudo contraste com a alta relevância e demanda de uma política prioritária que se aproxima de duas décadas devultosos investimentos. Estudos futuros podem se beneficiar dos condicionantes e indicadores das redes exitosas no país, das quais podem ser citados os estudos 'ELSA Brasil'46,47; 'ELSI Brasil'48; e 'ÉRICA'49. Mesmo configurada como 'rede de serviços', o peso científico e tecnológico de que dispõe a Rebrats a torna caso compulsório. Na interface política própria tem-se o exemplo do Observatório de Análise Política em Saúde (OAPS) ${ }^{50}$ e da Rede de Pesquisa em Atenção Primária à Saúde (APS).

Para concluir, o objetivo de conformação das redes confronta atualmente a necessidade de agenda estratégica que busque também adequar o campo disciplinar às expectativas futuras, incluindo cenários externos ao Sistema Nacional de Pós-Graduação. Para adquirir assento em um trem que ainda aceita passageiros, a SC terá que aprender a equilibrar com maior propriedade a balança entre os pesos político e científico de seu campo disciplinar.

\section{REFERÊNCIAS}

1. $\quad$ Paim JS, Almeida Filho $\mathrm{N}$ de. Saúde coletiva: uma "nova saúde pública" ou campo aberto a novos paradigmas? Rev Saúde Pública. 1998;32(4):299-316.

2. Mello GA. Quando os paradigmas mudam na saúde pública: o que muda na história? História Ciênc Saúde-Manguinhos. 2017;24(2):499-517.

3. Viana ALD, Elias PEM. Saúde e desenvolvimento. Ciênc Saúde Coletiva. 2007;12(supl):1765-77.

4. Souza LEPF Health, development and innovation: a contribution of the critical theory of technology to the discussion. Cad Saúde Pública. 2016;32(suppl 2):e00029615.

5. Buss PM, Chamas C, Faid M, Morel C. Development, health, and international policy: the research and innovation dimension. Cad Saúde Pública. 2016;32(suppl 2):e00046815.

6. Gadelha CAG, Costa LS. Saúde e desenvolvimento no Brasil: avanços e desafios. Rev Saúde Pública. 2012;46(suppl 1):13-20.

7. Schraiber L. Engajamento ético-político e construção teórica na produção científica do conhecimento em saúde coletiva. In: Baptista TWF, Azevedo CS, Machado CV, organizadores. Políticas, planejamento e gestão em saúde: abordagens e métodos de pesquisa. Rio de Janeiro: Fiocruz; 2015.

8. Paim JS, Teixeira CF. Política, planejamento e gestão em saúde: balanço do estado da arte. Rev Saúde Pública. 2006;40(spe):73-8.

9. Ilinca S, Hamer S, Botje D, Espin J, Mendes RV, Mueller J, et al. All you need to know about innovation in healthcare: The 10 best reads. Int J HealthcManag. 2012;5(4):193-202.

10. Wilson Center, Interfarma, Negri F. Novos caminhos para a inovação no Brasil. Washington, D.C: Wilson Center; 2018. 159 p.

11. Guimarães R. Pesquisa no Brasil: a reforma tardia. São Paulo em Perspect. 2002;16(4):41-7.

12. Guimarães R. Bases para uma política nacional de ciência, tecnologia e inovação em saúde. Ciênc Saúde Coletiva. 2004;9(2):375-87.

13. Zerhouni E. The NIH Roadmap. Science. 2003;302(5642):63-72.

14. Zerhouni EA. US biomedical research: basic, translational, and clinical sciences. J Am Med Assoc. 2005;294(11):1352-8.

15. Zerhouni EA. Translational and clinical science - time for a new vision. N Engl J Med. 2005;353(15):1621-3.

16. Guimarães R. Pesquisa translacional: uma interpretação. Ciênc Saúde Coletiva. 2013;18(6):1731-44. 
17. Guimarães R. Ciência, tecnologia e inovação: um paradoxo na reforma sanitária. In: Lima NT, organizador. Saúde e democracia: história e perspectivas do SUS. Rio de Janeiro: Fiocruz; 2005. p. 235-56.

18. Guimarães R. Pesquisa em saúde no Brasil: contexto e desafios. Rev Saúde Pública. 2006;40:3-10.

19. Ministério da Saúde (BR). Secretaria de Ciência, Tecnologia e Insumos Estratégicos. Departamento de Ciência e Tecnologia. Agenda nacional de prioridades de pesquisa em saúde [Internet]. 20 ed. Brasília: Editora do Ministério da Saúde; 2008 [citado 2012 jun 19]. 68 p. (Série B. Textos Básicos em Saúde). Disponível em: http://bvsms.saude.gov.br/bvs/publicacoes/AGENDA PORTUGUES MONTADO.pdf.

20. Tenório M, Mello GA, Viana ALD. Políticas de fomento à ciência, tecnologia e inovação em saúde no Brasil e o lugar da pesquisa clínica. Ciênc Saúde Coletiva. 2017;22(5):1441-54.

21. Viana AL d'Ávila, Silva HP, Ibañez N, Iozzi FL. A política de desenvolvimento produtivo da saúde e a capacitação dos laboratórios públicos nacionais. Cad Saúde Pública. 2016;32(suppl 2):e00188814.

22. Guimarães R. Desafios da pós-graduação em saúde humana no Brasil. Rev Saúde Pública. 2011;45(1):1-13.

23. Ministério da Saúde (BR). Secretaria de Ciência, Tecnologia e Insumos Estratégicos. Departamento de Ciência e Tecnologia. Relatório de gestão 2003: Departamento de Ciência e Tecnologia [Internet]. Brasília, DF: Ministério da Saúde; 2003 [citado 2019 ago 12]. Disponível em: http://dtr2001.saude.gov. br/editora/produtos/livros/popup/05 0285.htm.

24. Ministério da Saúde (BR). Chamada MCTI/CNPq/CT-Saúde/MS/SCTIE/Decit N o 41/2013 - Rede Nacional de Pesquisas sobre Política de Saúde: Conhecimento para Efetivação do Direito Universal à Saúde [Internet]. 2013 [citado 2019 ago 12]. Disponível em: http://www.cnpq.br/web/guest/ chamadaspublicas? $\mathrm{p} \mathrm{p}$ id=resultadosportlet WAR resultadoscnpqportlet INSTANCE 0ZaM\&filtro=abert as\&detalha=chamadaDivulgada\&idDivulgacao $=3722$.

25. Ministério da Saúde (BR). Portaria no 193, de 31 de janeiro de 2014. Institui a Rede Nacional de Pesquisa sobre Política de Saúde, composta pelas instituições de ensino e pesquisa da área da saúde e afins, envolvidas com os objetivos da Rede. [Internet]. 2014 [citado 2019 ago 12]. Disponível em: http://bvsms.saude.gov.br/bvs/saudelegis/gm/2014/prt0193 31 01 2014.html.

26. Ministério da Saúde (BR). Secretaria de Ciência, Tecnologia e Insumos Estratégicos. Departamento de Ciência e Tecnologia, organizador. Rede Nacional de Pesquisa Clínica. 2a. edição. Brasília, DF: Ministério da Saúde; 2010. 107 p. (Série B. Textos básicos de saúde).

27. Tenório M. A gestão de redes de pesquisa científica, tecnológica e de inovação em saúde no Brasil. [São Paulo]: Faculdade de Medicina da Universidade de São Paulo; 2016.

28. Tenório M, Mello GA, Viana ALD. Gestão e percepção de pesquisa em rede: uma visão a partir da Rede Nacional de Pesquisa Clínica em Hospitais de Ensino. Rev Eletron Comun Inf Inov Saúde. 2016 [citado 2019 ago 12];10(3):1-11. Disponível em https://www.reciis.icict.fiocruz.br/index.php/reciis/article/ view/1158/pdf1158.

29. Tenório M, Mena-Chalco JP, Mello GA, Viana ALD. Estrutura de pesquisa em rede: o caso da Rede Nacional de Pesquisas Clínicas em Hospitais de Ensino. P2P Inov. 2017;4(1):30-40.

30. Mena-Chalco JP, Cesar Junior RM. scriptLattes: an open-source knowledge extraction system from the Lattes platform. J Braz Comput Soc. 2009;15(4):31-9.

31. Mello GA. Estudo de caso sobre o Edital MCT/CNPq/MS-SCTIE-DECIT 25/2006: "Desenvolvimento de novas abordagens para o controle de Aedes aegypti, vetor de dengue, no Brasil". Relatório entregue ao Departamento de Ciência e Tecnologia do Ministério da Saúde (Decit-MS). 2012.

32. Ministério da Saúde (BR). Secretaria de Ciência, Tecnologia e Insumos Estratégicos. Departamento de Ciência e Tecnologia. Rede Nacional de Pesquisa Clínica do Brasil: respostas e redução da dependência estrangeira. Rev Saúde Pública. 2010;44(3):575-8.

33. Viana AL d'Ávila, Bousquat A, Ferreira MP, Cutrim MAB, Uchimura LYT, Fusaro ER, et al. Região e Redes: abordagem multidimensional e multinível para análise do processo de regionalização da saúde no Brasil. Rev Bras Saúde Materno Infant. 2017;17(suppl1):S7-16.

34. Minayo MCDS. A complexa dinâmica da divulgação científica. O caso da Revista Ciência \& Saúde Coletiva. Reciis. 2007;1(1):35-44. 
35. Ministério da Saúde (BR). Secretaria de Ciência, Tecnologia e Insumos Estratégicos. Departamento de Ciência e Tecnologia. Comunicação e informação do Decit: desafios para difusão e monitoramento das ações de fomento. Rev Saúde Pública. 2007;41(2):313-6.

36. PDTSP - Programa de Desenvolvimento Tecnológico em Saúde Pública [Internet]. [citado 21 nov 2018]. Disponível em: http://www.castelo.fiocruz.br/vpplr/pdtsp.php.

37. Wu L, Wang $D$, Evans JA. Large teams develop and small teams disrupt science and technology. Nature. 566(7744):378-82.

38. Braithwaite J, Churruca K, Ellis LA, Long J c, Clay-Williams R, Damen N, et al. Complexity science in healthcare - aspirations, approaches, applications and accomplishments: a white paper. 2017.

39. Johnson JD. Gestão de Redes de Conhecimento. São Paulo: Editora Senac; 2011. 440 p.

40. Metze K. Bureaucrats, researchers, editors, and the impact factor: a vicious circle that is detrimental to science. Clinics. 2010;65(10):937-40.

41. Marques F. Os limites do índice-h. Revista Pesquisa Fapesp. 2013;(maio):35-9.

42. Bardanachvili E. Quantidade não é qualidade. Radis Comun Em Saúde. 2015;maio (152):24-8.

43. Hicks D. The Leiden Manifesto for research metrics. Nature. 2015;520(7548):429-31.

44. Liu X, Bollen J, Nelson ML, Van de Sompel H. Co-authorship networks in the digital library research community. Inf Process Manag. 2005;41(6):1462-80.

45. Haddad EA, Mena-Chalco JP, Sidone O. Produção científica e redes de colaboração dos docentes vinculados aos programas de pós-graduação em Economia no Brasil. Estud Econômicos São Paulo. 2017;47(4):617-79.

46. Lotufo PA. Construção do Estudo Longitudinal de Saúde do Adulto (ELSA-Brasil). Rev Saúde Pública. 2013;47(suppl 2):3-9.

47. 47 ELSA Brasil: maior estudo epidemiológico da América Latina. Rev Saúde Pública [Internet]. 2009 fev [citado 2018 nov 26];43(1). Disponível em: http://www.scielo.br/scielo.php?script=sci arttext\&pid=S00 $3489102009000100028 \& \operatorname{lng}=p t \&$ tlng $=$ pt.

48. Lima-Costa MF, Andrade FB, Souza PRB, Neri AL, Duarte YA O, Castro-Costa E, et al. The Brazilian Longitudinal Study of Aging (ELSI-Brazil): Objectives and Design. Am J Epidemiol. 2018;187(7):134553.

49. Sichieri R, Cardoso MA. ERICA: Study of Cardiovascular Risk Factors in Adolescents. Rev Saúde Pública. 2016;50(suppl 1):1s.

50. Teixeira CF, Vilasbôas ALQ, Paim J. Mais que nunca, é preciso lutar... Saúde em Debate. 2018;42(spe2):5-6. 